\title{
Bruno Bureau
}

\section{L'autorité apostolique à travers les discours de l'Historia Apostolica d'Arator}

Parmi tous les auteurs que l'on rattache au genre de l'épopée biblique ${ }^{1}$, Arator est sans nul doute celui qui prend le plus de soin à exposer son projet et le sens qu'il va donner à sa lecture des Actes des Apôtres. Écrivant à l'évêque de Rome, Vigile, sans doute au printemps 544, au moment de lui remettre son Historia Apostolica ${ }^{2}$, il la présente en ces termes (Arat. Vig. 17-22) :

\footnotetext{
Sensibus ardor inest horum celebrare labores, quorum uoce fides obtinet orbis iter.

Versibus ergo canam quos Lucas rettulit Actus, historiamque sequens carmina uera loquar. Alternis resonabo modis quod littera pandit et res si qua mihi mystica corde datur ${ }^{3}$.
}

Si le texte de Luc est expressément nommé (Actus), le contenu du poème tel qu'il est décrit fait subir à ce matériau une double reconfiguration : le texte est envisagé sur le mode d'une forme d'épopée panégyrique (celebrare labores), mais surtout il est prévu comme un commentaire conforme à la pratique des sens multiples de l'Écriture $^{4}$. Le but de ce travail de commentaire du texte est bien d'actualiser, pour l'Église de Rome de 544, le message apostolique. On notera en effet le présent obtinet là où on attendrait obtinuit, signe que le travail d'évangélisation mené par les apôtres doit se poursuivre dans le présent et pour l'auditoire du poème lui-même qui se trouve ainsi placé dans la position des premiers auditeurs des apôtres.

Notre propos sera donc ici de montrer, à partir de quelques exemples, comment Arator dispose et recompose les discours des apôtres dans le but de construire une image de l'autorité apostolique dans l'Église conforme à la volonté de l'évêque de Rome Vigile. On se souviendra en effet que le poète écrit en un temps de controverses religieuses aiguës qui se doublent de plus de différends politiques très violents entre

1 Sur le genre lui-même voir par exemple Deproost (1997). Voir également Green (2006).

2 Sur les circonstances de cette remise, voir Arator (2017), p. xx-xxvii et 185-186. Sur les liens entre Arator et Vigile, voir Sotinel (1989).

3 Sur ce texte et en particulier sur le choix de la leçon resonabo, voir Arator (2017), p. 189-190. Voir également ibid. les antécédents de l'emploi de labores pour des héros chrétiens.

4 Le poète fait d'ailleurs lui-même une mise au point sur cette manière d'expliquer l'écriture en Arat. II.890-891 : Qui canit Ecclesiae tria dogmata saepius edit / historicum, morale sonans typicumque uolumen. On notera que cette méthode d'explication de l'Ecriture est explicitement rattachée à l'enseignement de la foi trinitaire. 
Goths et Byzantins, catholiques et ariens, monophysites, ariens et chalcédoniens ${ }^{5}$. Il est donc sans doute très important, voire crucial que le poète affirme l'autorité de Pierre et du Siège apostolique et donc la parfaite orthodoxie de celui-ci.

Pour mettre en évidence le travail considérable que le poète opère sur sa matière scripturaire, nous partirons de la place des discours dans l'économie du poème comparée à leur place dans les Actes, puis nous verrons, à partir de deux exemples, comment le poète reprend très vite la main sur son original et le recompose totalement à sa façon, avant de souligner, pour terminer, quelques points saillants de ce qu'Arator met sous le patronage de la catéchèse apostolique.

\section{La place des discours dans l'économie du poème : concentration, simplification, amplification et mise en scène épique.}

Dans son traitement des discours, Arator se livre à de très nombreuses modifications du matériau orignal des Actes qui touchent aux trois aspects fondamentaux de son projet : 1-donner un cadre épique à une geste qu'il veut représenter comme fondatrice, en peignant les apôtres comme les fondateurs de la nouvelle et parfaite cité de Rome, 2-éliminer tout ce qui pourrait détourner l'attention du lecteur de la contemplation des deux figures apostoliques de Pierre et Paul, et 3-développer certains discours pour faire apparaître clairement la dimension catéchétique de l'enseignement apostolique dans le contexte du $6^{\mathrm{e}}$ siècle.

Un bon exemple de la mise en place du cadre épique peut être donné par l'attaque des deux premiers discours de chaque apôtre. Dans les Actes, ces discours (1.16 et suiv. et 13.16 et suiv.) s'ouvrent de manière assez différente ${ }^{6}$, mais le poète n'a pas manqué de remarquer que dans l'annonce des deux discours le rédacteur des

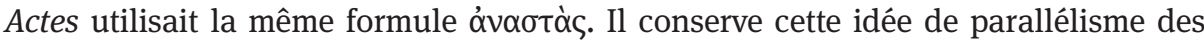
deux discours, mais la transporte sur la forme même de la parole, alors qu'il retravaille le geste de chaque apôtre pour lui donner un sens symbolique très fort.

Tout d'abord il rapproche les deux premières phrases des discours pour en faire deux facettes d'un même enseignement (Arat. I.83-84 et II. 43-44) :

...Nostis qua proditor amens

Mercedem sceleris soluit sibi;

5 Sur cette situation fort compliquée, voir Sotinel (1989) ; Sotinel (1992) ; Sotinel (2005). Voir également pour les questions théologiques Grillmeier (1993).

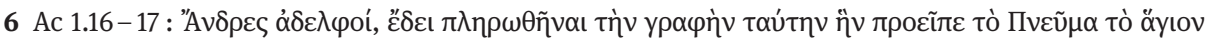

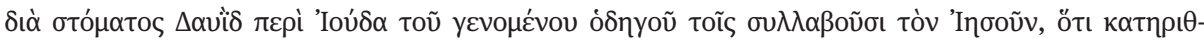

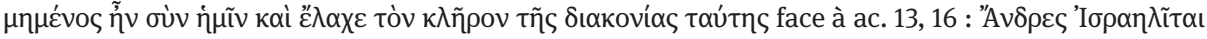

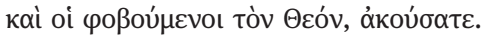


Nostis... patribus tellus Aegyptia nostris

Qua posuit feritate iugum

Ce déplacement en apparence sans importance tend clairement à souligner qu'il faut lire les paroles des deux apôtres comme strictement complémentaires, parce que leur fonction apostolique l'est également. Mais cette complémentarité est reportée à une lecture «politique» et historique de la prédication apostolique comme fondatrice de la nouvelle Rome, à travers les formules introductives qui ne doivent plus grandchose aux Actes. L'ouverture du livre 2 paraît la plus conforme à l'original ${ }^{7}$ et demeure assez sobre (Arat. II.40-42) :

Antiochi dictam de nomine uisitat urbem

Paulus et exemplo properat dare uerba cateruis

Quas synagoga tenet dextraque silentia mandans,...

mais le poète introduit une délicate référence épique à la fois par le choix du mot cateruis et par le renvoi à la figure de César dont Paul imite le geste (Lucan. I.298 ${ }^{8}$ ). $\mathrm{Au}$ contraire l'introduction du discours de Pierre donne lieu à la plus longue préparation du poème (Arat. I.69-83) :

Primus apostolico, parua de puppe uocatus, agmine Petrus erat, quo piscatore solebat Squamea turba capi. Subito de litore uisus, dum trahit, ipse trahi meruit. Piscatio Christi discipulum dignata rapit qui retia laxet humanum captura genus. Quae gesserat hamum, ad clauum est translata manus, quique aequoris imi ardebat madidas ad litora uertere praedas et spoliis implere ratem, melioribus undis nunc alia de parte leuat nec deserit artem, per latices sua lucra sequens. Cui tradidit Agnus quas passus saluauit oues, totumque per orbem hoc auget pastore gregem. Quo munere summus surgit et, insinuans diuina negotia, coram sic uenerandus ait:

Dans cet ensemble très complexe et qui rappelle les très longues introductions des discours épiques ${ }^{9}$, les derniers mots méritent toute notre attention dans la mesure où ils remotivent la figure d'Énée protecteur des siens, par un renvoi assez patent à Verg. Aen. VIII.69-71 :

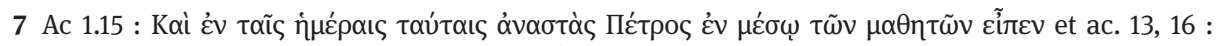

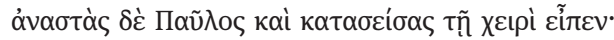

8 dextraque silentia iussit: sur le choix un peu étrange du personnage de Lucain qui n'est rien moins que sympathique, voir Bureau (1997), p. 182-183.

9 Voir par exemple Verg. Aen. XII.1-10. 
Surgit et aetherii spectans orientia solis

Lumina rite cauis undam de flumine palmis

Sustinet ac talis effundit ad aethera uoces:

«Nymphae, Laurentes nymphae, genus amnibus undest,

Tuque, o Thybri tuo genitor cum flumine sancto,

Accipite Aenean et tandem arcete periclis.

On peut donc ici parler d'un double geste fondateur d'autorité : d'un côté Pierre et Paul sont pourvus d'une forte coloration épique, et rapprochés de deux héros ouvertement dotés d'une valeur fondatrice, Énée et César, et de l'autre leur parole est présentée dès le début comme ce qui les constitue en tant que héros et assure à la fois leur lien protecteur à la communauté et l'articulation de leurs deux missions. En effet, la formule, plus ramassée pour Paul, et exempte de toute idée de primat largement affirmée pour Pierre ${ }^{10}$, prépare clairement l'image que le poète donne de l'autorité conjointe des apôtres en Arat. II.216-218 ${ }^{11}$ :

...gloria rerum

contulit ut Petro Paulum gerat ordo secundum, qui fundamentis manet architectus in illis.

Ainsi les dimensions ecclésiale, politique et théologique de l'autorité apostolique s'affirment dès le début de l'oeuvre pour culminer dans la louange finale ( Arat. II.1233-1245) :

\footnotetext{
Dignaque materies Petri Paulique coronae

Caesareas superare minas et in arce tyranni pandere iura poli, summumque in agone tribunal uincere, ne titulos paruus contingeret hostis.

Aegyptus mundi formam gerit. Inde uocari quae meruit, ducibus plebs est commissa duobus in quibus officium fraternus nexuit ortus. Idola tot Romae, mundo collecta subacto quae fuerant, tenebris obnoxia corda premebant. Liber et hic populus, quem uinxerat ante Pharao, Exuit Aegypti totidem ductoribus umbras, perque lauacra Dei, quae tunc maris egit imago, Vitae nactus iter caelestem repperit escam.
}

Cette représentation des apôtres conditionne en réalité tout le travail de recomposition du matériau scripturaire, et en particulier le choix des discours conservés ou éliminés, ainsi que la place même de la parole apostolique dans l'ensemble de l'œuvre.

10 Sur la lecture pétrinienne de l'ouverture du discours, voir Deproost (1990), p. 142-147.

11 Sur ce texte voir Deproost (1990), p. 189 et Bureau (1997), p. 129 et 293. 
En effet, de très nombreux éléments que nous jugeons, nous, fondamentaux pour la compréhension des Actes sont purement et simplement omis par Arator, ce qui le distingue par exemple du soin méticuleux que Juvencus mettait à décrire tous les épisodes de la vie de Jésus et Sédulius à décrire tous ses miracles. Cela est particulièrement vrai des discours.

Dans la partie des Actes des apôtres qui recouvre le livre 1 d'Arator, le rédacteur fait parler 23 personnages (parfois pour quelques mots) et Pierre prononce 38, $6 \%$ des paroles rapportées ; dans la seconde partie des Actes, parlent 40 personnages et Paul prononce $63 \%$ des paroles. Chez Arator, le livre 1 fait parler 5 personnages et Pierre prononce 69, $8 \%$ des paroles rapportées, tandis que le livre 2 fait parler 9 personnages et Paul prononce $84,3 \%$ des paroles ${ }^{12}$. Cette remarquable concentration sur les figures apostoliques s'accompagne évidemment de la disparition de bien d'autres locuteurs, la plus voyante étant celle d'Étienne dont le poète rapporte le martyre mais non les paroles.

On voit donc s'opérer dans cette sélection un centrage des discours sur la personne des apôtres Pierre et Paul, fût-ce au prix de l'élimination d'éléments essentiels du récit scripturaire. Mais cette tendance ne va pas sans une autre qui, derrière une apparente contradiction, confirme ce que nous venons de montrer : l'importance considérable que prennent les discours par rapport au reste du récit. Apparemment les discours sont moins importants chez Arator que dans les Actes (11,7\% du livre 1 et $23,4 \%$ du livre 2 contre $59,4 \%$ et $55,4 \%$ pour les deux parties respectives des Actes), mais cette lecture ne doit pas nous tromper car le récit chez Arator n'occupe qu'une partie très limitée de l'oeuvre, dont une bonne partie est dévolue au commentaire. Ainsi, si l'on ne prend en compte que les vers narratifs du livre 1 , soit environ 300 vers, les discours en occupent $42 \%$, ce qui se rapproche de la pratique des Actes.

On voit donc que le poète conserve à peu près la répartition entre discours et récits dans les Actes (en la réduisant un peu en éliminant des discours qu'il juge inutiles), mais que surtout il englobe l'ensemble dans un système commentatif qui recouvre environ $70 \%$ du texte et qui de ce fait constitue le poème plus comme une série d'homélies en vers que comme un récit proprement dit.

Ces tendances complexes ne sont cependant pas contradictoires, car le poète articule clairement la dimension épique, discursive et homilétique de son travail. La dimension épique lui sert à fournir un cadre à l'actualisation historico-politique du texte, ce qui fonde de manière renouvelée l'auctoritas de la parole apostolique. Le discours apparaît alors comme la pierre angulaire d'un enseignement que le poète va ensuite développer et préciser au gré de ses propres commentaires. La question de l'autorité se déplace donc légèrement pour se poser ainsi : comment le poète accrédite-t-il sa propre parole par la parole des apôtres ?

12 Pour le détail, voir Bureau (1997), p. 229-231 et Arator (2017), p. xxiv et suiv. 


\section{La manière de construire les discours en parallèle avec les discours des Actes, paraphrase exégèse et cathéchèse.}

Pour montrer le travail opéré par le poète pour «actualiser» son propos à l’Église de son temps, le discours d'adieu de Paul aux Anciens d'Asie fournit un excellent champ d'observation. Discours essentiel dans les Actes (20.18b-35), il l'est aussi dans le poème, dont il est le plus long morceau d'une seule pièce au style direct (Arat. II.831-879). On peut aborder le travail poétique sur ce discours de trois manières : la structure à la fois héritée et nouvelle que lui donne Arator, la manière dont il utilise les poètes antérieurs pour colorer son discours, et la théologie qu'il ajoute à la parole paulinienne originale.

La comparaison des deux structures montre un respect parfait par le poète de la macrostructure du discours paulinien, qui donne à penser à une stricte paraphrase de l'original. Les trois parties (rappel de la mission, évocation de la suite de la mission et recommandations finales) sont parfaitement conservées, mais le poète modifie considérablement les proportions en faveur de la partie centrale, celle qui concerne les suites de la mission. Or, si l'on observe le détail, le poète a considérablement remanié le discours en déplaçant plusieurs éléments essentiels, éliminant et ajoutant partout.

Les quatre ajouts sont évidemment les éléments-clé de la transformation, et ils relèvent clairement à la fois du rapport que le poète entretient avec la tradition poétique épique antérieure et du rapport qu'il entretient avec la tradition même de l'Église.

Le premier ajout, l'adresse solennelle, n'a rigoureusement aucun correspondant dans les Actes, mais en a plusieurs dans la tradition poétique antérieure, et, allusivement, dans la tradition paulinienne :

O delecta manus quae Christi militat armis!

O summo plebs nata Deo!

Le renvoi probable à l'univers épique passe ici par une formule chère à Silius Italicus, croisée avec un souvenir de l'éloge de Constantin par Optatien Porphyre ${ }^{13}$. Dans les deux cas, la parole apostolique se donne non comme une parole d'adieu, mais comme une exhortation au combat dans un monde romain désormais devenu chrétien et où la militia n'est plus celle du prince ou du roi terrestre mais du vrai roi du monde, le Christ. En même temps que le thème paulinien du combat spirituel se glisse, via une allusion à Paulin de Périgueux, celui de l'enfantement en Christ qui

13 Opt. 16.23-25: Omnis ab Arctois plaga finibus horrida Cauro / Pacis amat cana et comperta perennia iura, / Et tibi fida tuis semper bene militat armis, 
caractérise les saints ${ }^{14}$. Dès ce début, on observe donc comment le poète oriente la lecture du discours dans un sens qui n'est pas exclusivement, voire prioritairement celui de l'original.

Les deuxième et troisième ajouts, qui se suivent, soulignent une autre manière de repenser le texte des Actes et donc de le commenter en feignant de le paraphraser (Arat. II.839b-844) :

...Vos conuenit inde

usuram praestare piam cum uenerit Auctor, qui meriti discussor erit seruosque reposcet mensurae crementa suae. Mihi germina ferre sensibus ardor erat, quae passim credita sulcis sparsimus. At fructus tenues mala terra dolebit.

Plusieurs références évangéliques se croisent ici (Mt 25.14-30 et 13.3-23), et rattachent la parole apostolique à l'enseignement de Jésus, mais le plus intéressant n'est sans doute pas là, mais dans trois renvois de la parole apostolique à des éléments contemporains. Le premier et le plus voyant est le renvoi au poème lui-même à travers la reprise de la iunctura sensibus ardor qui se trouve aussi en Arat. Vig. 15-16 :

sensibus ardor inest horum celebrare labores,

quorum uoce fides obtinet orbis iter.

Cette reprise indique clairement que l'actualisation même de la parole de Paul se trouve dans le poème, et, par un mouvement inverse, que le poème est tout entier dépendant de la parole apostolique qui seule le justifie. Mais on peut aisément trouver un autre point de contact avec la réalité contemporaine, dans la lettre que Vigile adresse à Auxanius l'année précédant la récitation du poème, et où il parle de la charge de l'épiscopat, comme des talents confiés par Dieu aux bons serviteurs pour qu'ils les fassent fructifier (Epist. pontif. Vigil. ad Auxanium 3 [PL 69, 27]) :

superest ut Deus qui nos immeritos ineffabili misericordia honorem miserans pontificalem habere pro sua pietate concedit, donet et praemium, ut illud in nobis compleatur quod Christi Dei et Saluatoris nostri uerba testantur dicentis : «Euge serue bone et fidelis, quia in pauca fuisti fidelis supra multa te constituam "... sunt etiam quae per Dei gratiam... ad facienda bona hortentur exempla. Si enim decessoris tui illa quae a sede apostolica de fundamento petrae dominicae doctrinae bona suscipiens actibus exaequauit, imitari uolueris, et a sedis apostolicae in nullo deuiaueris constitutis.

Ainsi, la charge épiscopale et les devoirs qui s’y rattachent sont inscrits dans les mêmes termes dans la parole apostolique et dans la parole du successeur de Pierre et

14 Paul. Petric. Mart. I.232-234 : O tanto partu felix enixaque natum, / Per quem nata Deo est, unoque et mater et infans / Facta puerperio! 
les normes qu'il édicte sont comme des normes édictées par l'apôtre lui-même. Le poète marque ainsi une continuité parfaite entre l'enseignement apostolique et celui de l'évêque de Rome.

Il reste à voir, avec le dernier ajout, comment le poète utilise Paul lui-même pour compléter l'autorité du discours de son personnage de paroles non pas prononcées par lui en cette circonstance, mais qui relèvent de sa doctrine et qui donc, conformément aux canons de l'historiographie antique, pourraient lui être ici attribuées (Arat. II.871-879) :

Assuescite gazas
In lucis proferre uias operumque locare
Thesauros in sede poli; nil proderit aurum
Defossis quaesisse locis, si claudat auarus
Quod celabat humus; quod non in paupere surgit,
In tellure iacet caecoque reuoluitur antro
Obscura peccante manu; laxate supernis
Corda, precor, monitis, neque respuat ullus egeno
Tecta parare gregi, quo suscipit hospite Christum.

Tout le passage se fonde évidemment sur une lecture de $1 \mathrm{Tm}$ 6.17-19, où l'apôtre disait :

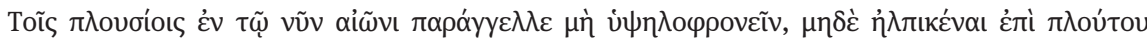

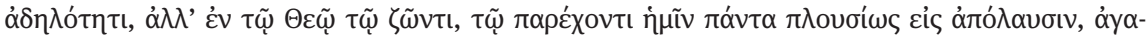

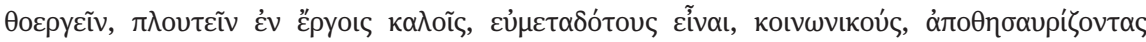

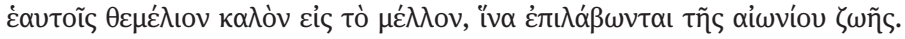

Mais il ne le fait pas exclusivement car le poète reprend l'image évangélique des trésors dans le ciel (Mt. 6.20), au point que les références évangéliques et pauliniennes se croisent sans cesse et s'interpénètrent de façon à rendre difficilement identifiable le passage de l'un à l'autre. Ce faisant, le poète associe sans cesse l'autorité apostolique et l'autorité évangélique, mais il le fait dans la lumière de la tradition de l'Église. Tout le développement est de fait en grande partie tributaire d'une homélie d'Augustin sur la fête de Pierre et Paul (Aug. serm. 296.9) :

quid recusas uapulare multis, o serue sciens uoluntatem domini tui, et faciens digna plagis? dicitur tibi - ecce una uoluntas domini tui: thesaurizate uobis thesaurum in caelo, ubi neque tinea neque aerugo neque comestura exterminat, et ubi fures non effodiunt, neque furantur. tu in terra, ille in caelo, dicens tibi: mihi da, ibi habeto thesaurum, ubi ego sum custos, mitte ante te: quid seruas? quod custodit christus, numquid tollit gothus? tu contra, prudentior scilicet et sapientior domino tuo, non nisi in terra thesaurizare uis. sed cognouisti uoluntatem domini tui, sursum te condere uoluit ille: ergo, in terra condens, paratus esto uapulare multis.

L'utilisation de l'enseignement augustinien achève ici le processus de création de l'autorité. Paul et la tradition catholique parlent de la même voix, et cette voix est en 
amont celle de l'évangile et en aval celle du siège apostolique voulu par Dieu pour conserver intact le dépôt de la vraie foi.

On comprend alors l'importance que revêtent les discours dans l'Historia Apostolica et la nécessité de leur recomposition. Ils deviennent le lieu où se manifeste de la manière la plus immédiate la continuité de la tradition évangélique et apostolique dans la foi de l'Église romaine, seule apte à énoncer la vérité de la foi dans la succession pétrinienne.

On peut donc alors observer les éléments que le poète insère sous cette autorité particulière et qu'il ajoute aux Actes pour en actualiser le propos. Ce sera le but de notre troisième et dernière partie que nous centrerons sur les discours de Pierre.

\section{Les insertions théologiques dans les discours, une catéchèse pour la Rome du $6^{\mathrm{e}}$ siècle.}

Pierre parle bien moins que Paul, mais, outre son discours d'ouverture sur lequel, faute de temps nous ne reviendrons pas, il prononce trois discours importants, quoique brefs, deux au livre 1 (devant les Anciens du Temple sur la valeur de son témoignage, Arat. I.297-301, et devant le magicien Simon, Arat. I.634-642) et un au livre 2, lorsque le poète lui fait, à la place de Jacques dans les Actes, régler la question des obligations légales auxquelles sont soumis les chrétiens (Arat. II.262273). On notera immédiatement que le poète élimine des discours extrêmement importants de Pierre, comme par exemple le discours qu'il tient au peuple après la guérison du boiteux de la Belle Porte (ac 3.12-26), celui à Corneille (ac 10.28 - 43), ou celui qu'il fait devant l'Église pour justifier le baptême des païens (ac 11, 5-17). Il faut donc évidemment expliquer pourquoi il conserve ceux-là et quelle forme d'autorité ils portent.

Le premier discours insiste clairement à la fois sur la nouveauté inouïe du message chrétien et son caractère salvateur (Arat. I.297-301 = ac 4.8-12) :

\footnotetext{
« Non hunc reticebimus » inquit

« quo remeat praestante salus qui iure creantis

infectum suppleuit opus, solidumque decorem

pars reparet quam iussa nouant. Hic membra redemptor

saucia restituit qui mortua surgere cedit ».
}

Ce discours est surtout remarquable par l'élimination de plusieurs éléments le rattachant à son contexte juif, que ce soit l'apostrophe aux Juifs, la mention de Nazaréen ou la citation de ps 117.22, pourtant très utilisé dans l'argumentation christologique $^{15}$. Mais ce qui est surtout remarquable est l'ouverture du discours non

15 Le texte original disait (ac 4.8-12) : «Chefs du peuple et anciens, nous sommes interrogés aujourd'hui pour avoir fait du bien à un infirme, et l'on nous demande comment cet homme a été 
reticebimus, qui impose la figure de Pierre, et de son successeur, comme celui qui ne peut pas ne pas annoncer la vérité de la foi, quoi qu'il doive lui en coûter. De plus, l'argumentaire de Pierre se teinte de fortes colorations pauliniennes, soulignant ainsi que d'un apôtre à l'autre la continuité doctrinale est totale. Arator introduit en effet pour évoquer le sacrifice du Christ une nette référence à Ph 3.20-21 où l'apôtre disait :

«Mais nous, nous avons notre citoyenneté dans les cieux, d'où nous attendons comme sauveur le Seigneur Jésus Christ, lui qui transformera nos pauvres corps à l'image de son corps glorieux, avec la puissance active qui le rend même capable de tout mettre sous son pouvoir».

Ainsi l'enseignement apostolique apparaît comme une doctrine unifiée et cohérente, ce qui en renforce évidemment l'autorité et le choix comme locuteur de Pierre, le disciple choisi par le Seigneur lui-même pour "affermir ses frères» impose immédiatement ces paroles comme la norme absolue de la foi.

Le deuxième discours adressé à Simon le magicien est encore plus clairement marqué par le souci de manifester la tradition de l'Église, mais cette fois dans un contexte de discipline morale (Arat. I.634-642 = ac 8.20) :

«Quisnam te, perdite,» dixit

«mouit ad ista furor, Domini quod gratia donat, ut uenale putes? Sensu hoc, non sumitur auro, nec licet ut caelum corrupta pecunia quaerat, quam terrae scrutator amat. Tibi nulla profecto hac superest in sorte quies nec ad ista uenire tu poteris, pollute dolis, qui cordis amari felle tumens aliena petis. Namque atria mentis Spiritus illa subit quae simplicitate nitescunt».

Le premier élément remarquable est évidemment que le poète supprime toute mention d'un possible pardon pour le personnage qui est réduit à sa faute ${ }^{16}$. Or cette faute elle-même est assez nettement déplacée, d'un simple désir d'argent, à la corruption même du message chrétien qui professe la gratuité du don de Dieu, puisque

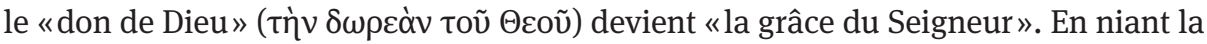

sauvé. Sachez-le donc, vous tous, ainsi que tout le peuple d'Israël : c'est par le nom de Jésus le Nazaréen, lui que vous avez crucifié mais que Dieu a ressuscité d'entre les morts, c'est par lui que cet homme se trouve là, devant vous, bien portant. Ce Jésus est la pierre méprisée de vous, les bâtisseurs, mais devenue la pierre d'angle. En nul autre que lui, il n'y a de salut, car, sous le ciel, aucun autre nom n'est donné aux hommes, qui puisse nous sauver».

16 Ce pardon possible était explicite dans l'original qui disait (ac 8.20-23) : «Périsse ton argent, et toi avec, puisque tu as estimé pouvoir acheter le don de Dieu à prix d'argent! Tu n'as aucune part, aucun droit, en ce domaine, car devant Dieu ton cœur manque de droiture. Détourne-toi donc de ce mal que tu veux faire, et prie le Seigneur : il te pardonnera peut-être cette pensée que tu as dans le cœur. Car je le vois bien : tu es plein d'aigreur amère, tu es enchaîné dans l'injustice». 
gratuité de la Grâce, Simon se condamne lui-même, et n'a plus de place pour le pardon. Ainsi la simonie ou l'amour des richesses par les clercs deviennent une faute contre la Grâce même de Dieu et donc non une faute seulement morale, mais une forme de déviance dans la foi. Ici évidemment le poète se souvient, pour faire parler le chef de l'Église, d'Aug. in psalm. 30.2.14, 21 :

non est tibi pars neque sors in hac fide; id est, non pertines ad istam gratiam, quam gratis omnes accipimus, quia pecunia te putas emere quod gratis datur. ex eo autem quod gratis datur, sors uocatur: non est tibi pars neque sors in hac fide. ista dixi, ne expauesceremus quod ait: in manibus tuis sortes meae. quae sunt enim sortes? hereditas ecclesiae.

C'est clairement cet emprunt homilétique qui opère le transfert de la réflexion morale à la réflexion ecclésiale et théologique, qui, mise dans la bouche de Pierre, prend immédiatement la valeur d'une norme intangible pour l'Église.

Le dernier discours de Pierre peut alors affirmer clairement l'autorité de l'apôtre et de son successeur dans les prescriptions légales qui constituent l'Église. Tout l'enjeu ici réside dans un double déplacement du texte original ${ }^{17}$ pour aboutir au discours de Pierre tel que le recompose le poète (Arat. II.262-273) :

\begin{abstract}
Cernitis Aeternum, saeclis memorata uetustis quae populo docili cecinerunt ore Prophetae, in nobis complesse Deum, qui maluit emptor omnibus esse salus, nullum discernere passus in pretio quo uita redit. Mihi iussit apertam gentibus hanc monstrare uiam. Quid uota morari tardarique iuuat, quidue haec aenigmata cana misceri cum luce noua ? Quos gratia purgat, ut ueniant, lex nulla uetat. Caelestis amoris materia est festina fides. Hanc Christus adoptat, hanc facit esse suam. Qua quisque merebitur uti, circumcisus adest et iure renascitur undis.
\end{abstract}

Le premier élément essentiel est extérieur à ce discours, mais fondamental pour la compréhension de l'épisode tel que le livre le poème. La décision, qui dans les Actes revient essentiellement à Jacques (ac 15.13-22), est ici le seul fait de Pierre, puisque Jacques n'apparaît même pas et que son discours n'est pas rapporté, le poète passant directement à la rédaction de la lettre pour les Églises. C'est donc Pierre, et Pierre seul, qui donne la décision et il est difficile de ne pas voir dans cette adaptation une

17 Ac 15. 7b-11: «Frères, vous savez bien comment Dieu, dans les premiers temps, a manifesté son choix parmi vous : c'est par ma bouche que les païens ont entendu la parole de l'Évangile et sont venus à la foi. Dieu, qui connaît les cœurs, leur a rendu témoignage en leur donnant l'Esprit Saint tout comme à nous ; sans faire aucune distinction entre eux et nous, il a purifié leurs cœurs par la foi. Maintenant, pourquoi donc mettez-vous Dieu à l'épreuve en plaçant sur la nuque des disciples un joug que nos pères et nous-mêmes n'avons pas eu la force de porter ? Oui, nous le croyons, c'est par la grâce du Seigneur Jésus que nous sommes sauvés, de la même manière qu’eux». 
allusion directe à l'autorité souveraine de son successeur pour toutes les questions de discipline ecclésiastique. D’ailleurs la reprise du récit est sans appel sur ce point (Arat. II.274) :

pastorem statuere sequi.

Le deuxième élément important est celui de la recomposition du plan du discours pour lui donner une structure de parole d'autorité que n'a pas l'original qui se contente de soumettre une question à la communauté. Le propos de Pierre est clairement en trois parties : l'apôtre commence par poser comme une évidence issue de l'Écriture l'appel universel au salut, puis il se pose en figure d'autorité, avant de résoudre lui-même le problème posé, en affirmant la non-nécessité de la circoncision. Or dans l'original, les deux premières parties sont imbriquées et Pierre se place en Juif qui a été choisi pour étendre le salut des Juifs aux Nations. C'est également en Juif qu'il interroge la pratique légale, avant de donner sa conviction sur la question posée. Toute la fin, très clairement normative et dogmatique dans le poème, n'existe purement et simplement pas dans l'original. On assiste donc ici, à travers une apparente fidélité à l'original, à une transposition complète du message apostolique dans le contexte de l'Église contemporaine au poète, comme si les siècles qui séparent les apôtres d'Arator étaient abolis.

La question de l'autorité apostolique apparaît donc comme le cœur du travail de recomposition que le poète fait subir aux discours des Actes des apôtres. Mais cette autorité n'est pas envisagée d'un point de vue historique (comment s'exerçait l'autorité dans les premiers temps de l'Église?), mais d'un point de vue doctrinal et disciplinaire, en lien avec l'Église de son temps. Il y a donc clairement à l'œuvre un procédé d'actualisation du texte pour le faire résonner dans le contexte politicoreligieux du $6^{\mathrm{e}}$ siècle romain.

En jouant sur les éléments épiques pour construire une figure héroïque et fondatrice des deux apôtres Pierre et Paul, le poète légitime une forme de translatio imperii de la Rome des Césars à la celle des successeurs de Pierre qui seuls gardent l'héritage de l'empire universel à travers l'universalité de l'Église.

Mais, en concentrant les discours et l'attention du public sur la figure des deux apôtres romains, Pierre et Paul, le poète fait dévier le contenu des Actes vers ce que P.A. Deproost a appelé une vision pétrinienne et romaine du texte, qui en modifie radicalement à la fois le sens et la portée théologique. C'est ce que confirme la technique de transformation des discours où Arator mélange avec virtuosité références évangéliques, pauliniennes et patristiques, pour faire tenir à ses apôtres le discours de la tradition catholique romaine, face à toute forme d'hérésie ou de contestation de l'autorité romaine.

On voit donc que le terme de paraphrase en vers ne s'applique absolument pas à ce texte qui est en réalité une construction autonome, se fondant sur les Actes des 
apôtres, mais dont le but est bien lié aux nécessités et aux combats de l'Église romaine de Vigile. Le poète qui est en réalité plus un exégète qu'un conteur parachève ainsi l'évolution du genre de l'épopée biblique, vers une forme versifiée d'homilétique.

\section{Bibliographie}

Arator (2017): Histoire apostolique, texte établi et traduit par B. Bureau \& P.-A. Deproost (trad.), Paris, France, les Belles lettres.

Bureau (1997): Bruno Bureau, Lettre et sens mystique dans l'«Historia apostolica» d'Arator: exégèse et épopée, Paris, France, Institut d'études augustiniennes.

Deproost (1990): Paul-Augustin Deproost, L'apôtre Pierre dans une épopée du Vie siècle: l'«Historia apostolica» d'Arator, Paris, France, Institut d'études augustiniennes.

Deproost (1997): Paul-Augustin Deproost, «L'épopée biblique en langue latine. Essai de définition d'un genre littéraire», Latomus 56, p. 14-39.

Green (2006): Roger P.H. Green, Latin epics of the New Testament: Juvencus, Sedulius, Arator, Oxford ; New York, Oxford University Pr.

Grillmeier (1993): Alois Grillmeier, Le Christ dans la tradition chrétienne. Tome II/2, L’Église de Constantinople au Vle siècle, Pascale-Dominique (trad.), Paris, France, les Éd. du Cerf.

Sotinel (1989): Claire Sotinel, «Arator, un poète au service de la politique du pape Vigile?», Mélanges L'École Fr. Rome Antiq., Cl, p. 805-820.

Sotinel (1992): Claire Sotinel, «Autorité pontificale et pouvoir impérial sous le règne de Justinien: le pape Vigile», in: Mélanges L'École Fr. Rome Antiq., CIV, p. 439-463.

Sotinel (2005): Claire Sotinel, «Emperors and popes in the sixth century: the western view», M.Mass, éd. Camb. Companion Age Justinian, p. 267-290. 
